\title{
Efficacy of Different Entomopathogenic Fungi against Mango Hoppers in Middle Gujarat
}

\author{
B. L. Raghunandan, T. B. Kapadiya, N. B. Patel*, N. M. Patel and D. M. Mehta \\ AICRP on Biological Control of Crop Pests, Anand Agricultural University, \\ Anand (Gujarat), India \\ *Corresponding author
}

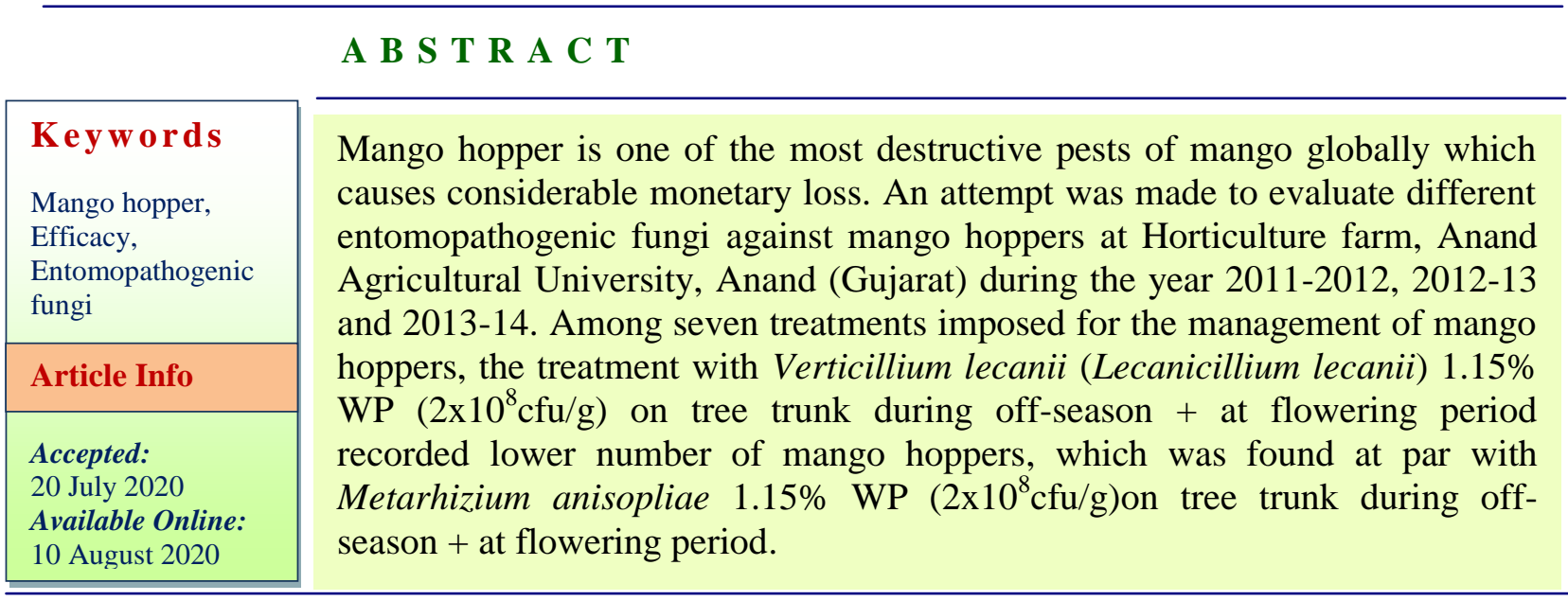

\section{Introduction}

Mango (Mangifera indica Linn.) is native of Indo-Burma region andmost ancient among the tropical fruits. Mangois considered as the "King of fruits" due to its attractive fragrance, delicious taste, excellent flavour, nutritional richness (Vitamin $\mathrm{A}$ and $\mathrm{C}$ ) and health promoting qualities. Among the pests that infesting mango, the cicadellids (jassids) popularly known as 'Mango hoppers' are economically important (Gangolly et al., 1957). Mango hopper, Amritodus atkinsoni (Lethierry) mainly inhabits the trunk region and new vegetative flush, while two
Idioscopus species, viz., Idioscopus clypealis (Lethierry) and Idioscopus nitidulus (Walker) infest during flowering season, which cause yield loss up to $50-60 \%$ in case of severe infestation (Tandonand Verghese,1985 and Gundappa, et al., 2014). Both the nymphs and adults of the hopper mainly suck the sap from tender shoots, leaves and inflorescence, which causes panicles shrivel, turn brown and finally fall off (Das, et al., 1969). Hoppers also excrete honey dew which causes sooty mould on leaf surface, interferes with the photosynthetic activity of the plant and finally affect the quality of fruit production. 
As regard control, using of numbers of broadspectrum insecticides is harmful to natural enemies on the crop. In addition to this, it creates environmental pollution and leaves pesticides residue on fruits. Pesticidal residues on mangoes affect export there by leading to financial losses. To overcome these problems, there is a need to develop the management techniques which are ecofriendly and safer to natural enemies. An important mechanism for mango hopper control should be the use of fungal entomopathogens. Therefore, the present study on evaluation of biological control agents against mango hoppers was carried out.

\section{Materials and Methods}

A study one valuation of entomopathogenic fungi against mango hoppers was carried out at Horticulture farm, AAU, Anand during 2011-12, 2012-13 and 2013-14. The experiment was laid out with cultivar, Langra in Completely Randomized Design (CRD) was followed with three repetitions. The experiment consists of 7 treatments (Table 1) and each tree was considered as one repetition. One off-season spray was given on tree trunk during the month of November and three sprays were given on foliage during flowering stage at fortnightly interval with the initiation of pest. Observations on number of mango hoppers were recorded before imposing the treatment as well as 7 and 14 days after treatment. Ten twigs-panicles (approx. $15 \mathrm{~cm}$ length) from each tree were observed and number of hoppers per twig was recorded.

The data obtained from the experiments were subjected to square root transformation and subjected to ANOVA analysis. Significant differences among the means of different treatments were tested using Duncan's new multiple range test (DNMRT)

\section{Results and Discussion}

The year wise data on bio-efficacy of different biocontrol agents against hoppers infesting mango are presented hereunder:

\section{Year 2011-12}

The data (Table 2) on population of mango hopper recorded before spray showed nonsignificant difference among different treatments indicated that the hoppers population was uniformly distributed in all the experimental trees.

The data of pooled over periods and sprays for the first year (rabi, 2011-12) revealed significant reduction in hoppers population in all the treated trees compared to untreated control. The reduction in hoppers population was significantly higher in treatment of imidacloprid 17.8 SL applied on tree trunk during off-season + one spray of the same applied at flowering stage (4.56 hoppers/ twig) than rest of the treatments. However, in case of bio-pesticidal treatments, trees sprayed with either V. lecanii (5.90 hoppers/ twig) or M. anisopliae (6.16 hoppers/ twig) on tree trunk during off-season + at flowering stage also proved effective treatment and stood next to the chemical treatment. Whereas, application of entomopathogenic fungi only on the tree trunk during off-season proved inferior against mango hoppers.

\section{Year 2012-13}

Data on hoppers population recorded prior and 7 and 14 days after each spray in different treatments during rabi, 2012-13 are presented in Table 3. Data indicated that the hoppers population was uniformly distributed in all the experimental trees as it evident from the observations recorded before imposing of spray. 
Table.1 Details of entomopathogenic fungi used against mango hoppers

\begin{tabular}{|c|l|c|c|}
\hline \multicolumn{1}{|c|}{ Treatments } & Concentration & $\begin{array}{c}\text { Quantity (ml or } \mathbf{~ g}) / \\
\text { 10 litre water }\end{array}$ \\
\hline $\mathbf{T}_{\mathbf{1}}$ & $\begin{array}{l}\text { Application of Metarhiziumanisopliae1.15\% WP on tree } \\
\text { trunk during off-season + at flowering period }\end{array}$ & $2 \times 10^{8} \mathrm{cfu} / \mathrm{g}$ & 40 \\
\hline $\mathbf{T}_{\mathbf{2}}$ & $\begin{array}{l}\text { Application of M. anisopliae1.15\% WP on tree trunk during } \\
\text { off-season }\end{array}$ & $2 \times 10^{8} \mathrm{cfu} / \mathrm{g}$ & 40 \\
\hline $\mathbf{T}_{\mathbf{3}}$ & $\begin{array}{l}\text { Application of Verticilliumlecanii1.15\% } \\
\text { WP(Lecanicilliumlecanii) on tree trunk during off-season + } \\
\text { at flowering period }\end{array}$ & $2 \times 10^{8} \mathrm{cfu} / \mathrm{g}$ & 40 \\
\hline $\mathbf{T}_{\mathbf{4}}$ & $\begin{array}{l}\text { Application of V.lecanii1.15\% WP(L. Lecanii) on tree trunk } \\
\text { during off-season }\end{array}$ & $2 \times 10^{8} \mathrm{cfu} / \mathrm{g}$ & 3 \\
\hline $\mathbf{T}_{\mathbf{5}}$ & $\begin{array}{l}\text { One spray of imidacloprid 17.8SL (26.7 ga.i/ha) on tree } \\
\text { trunk during off-season }\end{array}$ & $0.005 \%$ & 3 \\
\hline $\mathbf{T}_{\mathbf{6}}$ & $\begin{array}{l}\text { One spray of imidacloprid 17.8SL (26.7 ga.i/ha) on tree } \\
\text { trunk during off-season + at flowering period }\end{array}$ & $0.005 \%$ & 3 \\
\hline $\mathbf{T}_{\mathbf{7}}$ & \begin{tabular}{l} 
Untreated control \\
\hline
\end{tabular} & - & - \\
\hline
\end{tabular}

Table.2 Effectiveness of different entomopathogenic fungi against mango hoppers (2011-12)

\begin{tabular}{|c|c|c|c|c|c|c|c|c|}
\hline \multirow[t]{3}{*}{ Treatments } & \multicolumn{8}{|c|}{ No. of mango hoppers/twig } \\
\hline & \multirow{2}{*}{$\begin{array}{l}\text { Before } \\
\text { spray }\end{array}$} & \multicolumn{2}{|c|}{$1^{\text {st }}$ Spray } & \multicolumn{2}{|c|}{$2^{\text {nd }}$ Spray } & \multicolumn{2}{|c|}{$3^{\text {rd }}$ Spray } & \multirow{2}{*}{$\begin{array}{l}\text { Pooled over } \\
\text { periods over } \\
\text { sprays }\end{array}$} \\
\hline & & $\begin{array}{c}7 \\
\text { DAS }\end{array}$ & $\begin{array}{c}14 \\
\text { DAS }\end{array}$ & 7 DAS & $\begin{array}{c}14 \\
\text { DAS }\end{array}$ & $\begin{array}{c}7 \\
\text { DAS }\end{array}$ & $\begin{array}{c}14 \\
\text { DAS }\end{array}$ & \\
\hline $\mathbf{T}_{1}$ & $\begin{array}{c}3.44 * \\
(11.33)\end{array}$ & $\begin{array}{l}2.85 \\
(7.62)\end{array}$ & $\begin{array}{c}3.07 \\
(8.92)\end{array}$ & $\begin{array}{c}2.38 \\
(5.16)\end{array}$ & $\begin{array}{c}2.70 \\
(6.79)\end{array}$ & $\begin{array}{c}2.18 \\
(4.25)\end{array}$ & $\begin{array}{c}2.30 \\
(4.80)\end{array}$ & $\begin{array}{c}2.58 \\
(6.16)\end{array}$ \\
\hline $\mathbf{T}_{2}$ & $\begin{array}{c}3.31 \\
(10.46)\end{array}$ & $\begin{array}{c}3.58 \\
(12.32)\end{array}$ & $\begin{array}{c}3.75 \\
(13.56)\end{array}$ & $\begin{array}{c}3.84 \\
(14.25)\end{array}$ & $\begin{array}{c}3.80 \\
(13.94)\end{array}$ & $\begin{array}{c}3.80 \\
(13.94)\end{array}$ & $\begin{array}{c}3.91 \\
(14.79)\end{array}$ & $\begin{array}{c}3.79 \\
(13.86)\end{array}$ \\
\hline $\mathbf{T}_{3}$ & $\begin{array}{c}3.42 \\
(11.20)\end{array}$ & $\begin{array}{c}2.91 \\
(7.97)\end{array}$ & $\begin{array}{c}2.95 \\
(8.20)\end{array}$ & $\begin{array}{c}2.53 \\
(5.90)\end{array}$ & $\begin{array}{c}2.56 \\
(6.05)\end{array}$ & $\begin{array}{c}2.55 \\
(6.00)\end{array}$ & $\begin{array}{c}2.15 \\
(4.12)\end{array}$ & $\begin{array}{c}2.53 \\
(5.90)\end{array}$ \\
\hline $\mathbf{T}_{4}$ & $\begin{array}{c}3.51 \\
(11.82)\end{array}$ & $\begin{array}{c}3.53 \\
(11.96)\end{array}$ & $\begin{array}{c}3.61 \\
(12.53)\end{array}$ & $\begin{array}{c}3.58 \\
(12.32)\end{array}$ & $\begin{array}{c}3.71 \\
(13.26)\end{array}$ & $\begin{array}{c}3.71 \\
(13.26)\end{array}$ & $\begin{array}{c}3.83 \\
(14.17)\end{array}$ & $\begin{array}{c}3.67 \\
(12.97)\end{array}$ \\
\hline $\mathbf{T}_{5}$ & $\begin{array}{c}3.17 \\
(9.55)\end{array}$ & $\begin{array}{c}3.39 \\
(10.99)\end{array}$ & $\begin{array}{c}3.69 \\
(13.12)\end{array}$ & $\begin{array}{c}3.72 \\
(13.34)\end{array}$ & $\begin{array}{c}3.63 \\
(12.68)\end{array}$ & $\begin{array}{c}3.63 \\
(12.68)\end{array}$ & $\begin{array}{c}3.75 \\
(13.56)\end{array}$ & $\begin{array}{c}3.64 \\
(12.75)\end{array}$ \\
\hline $\mathbf{T}_{6}$ & $\begin{array}{c}3.24 \\
(10.00)\end{array}$ & $\begin{array}{c}2.61 \\
(6.31)\end{array}$ & $\begin{array}{c}2.85 \\
(7.62)\end{array}$ & $\begin{array}{c}2.10 \\
(3.91)\end{array}$ & $\begin{array}{c}2.33 \\
(4.93)\end{array}$ & $\begin{array}{c}2.33 \\
(4.93)\end{array}$ & $\begin{array}{c}1.87 \\
(3.00)\end{array}$ & $\begin{array}{c}2.25 \\
(4.56)\end{array}$ \\
\hline $\mathbf{T}_{7}$ & $\begin{array}{c}3.65 \\
(12.82)\end{array}$ & $\begin{array}{c}3.81 \\
(14.02)\end{array}$ & $\begin{array}{c}3.91 \\
(14.79)\end{array}$ & $\begin{array}{c}3.82 \\
(14.09)\end{array}$ & $\begin{array}{c}3.94 \\
(15.02)\end{array}$ & $\begin{array}{c}3.94 \\
(15.02)\end{array}$ & $\begin{array}{c}4.08 \\
(16.15)\end{array}$ & $\begin{array}{c}3.93 \\
(14.94)\end{array}$ \\
\hline S. $\mathbf{E m} \pm \mathbf{T}$ & 0.28 & 0.22 & 0.24 & 0.21 & 0.22 & 0.17 & 0.22 & 0.10 \\
\hline $\mathbf{Y} \times \mathbf{T}$ & - & - & - & - & - & - & - & 0.21 \\
\hline C.D. at $5 \% \mathrm{~T}$ & NS & 0.66 & 0.71 & 0.63 & 0.66 & 0.52 & 0.66 & 0.27 \\
\hline $\mathbf{Y} \times \mathbf{T}$ & - & - & - & - & - & - & - & NS \\
\hline C. V. $(\%)$ & 14.21 & 11.71 & 11.95 & 11.47 & 11.55 & 9.79 & 12.11 & 11.49 \\
\hline \multicolumn{7}{|c|}{ * Figures in parentheses are retransformed values; those outsidoare } & \multicolumn{2}{|c|}{ transformed values } \\
\hline
\end{tabular}


Table.3 Effectiveness of different entomopathogenic fungi against mango hoppers (2012-13)

\begin{tabular}{|c|c|c|c|c|c|c|c|c|}
\hline \multirow[t]{3}{*}{ Treatments } & \multicolumn{8}{|c|}{ No. of mango hoppers/twig } \\
\hline & \multirow{2}{*}{$\begin{array}{l}\text { Before } \\
\text { spray }\end{array}$} & \multicolumn{2}{|c|}{$\mathbf{1}^{\text {st }}$ Spray } & \multicolumn{2}{|c|}{$2^{\text {nd }}$ Spray } & \multicolumn{2}{|c|}{$3^{\text {rd }}$ Spray } & \multirow{2}{*}{$\begin{array}{l}\text { Pooled over } \\
\text { period over } \\
\text { sprays }\end{array}$} \\
\hline & & 7 DAS & $\begin{array}{c}14 \\
\text { DAS }\end{array}$ & 7 DAS & $\begin{array}{c}14 \\
\text { DAS }\end{array}$ & 7 DAS & 14 DAS & \\
\hline \multirow[t]{2}{*}{$\mathbf{T}_{1}$} & $3.70^{*}$ & 3.12 & 3.43 & 2.78 & 2.98 & 2.66 & 2.69 & 2.94 \\
\hline & $(13.19)$ & $(9.23)$ & (11.26) & $(7.23)$ & $(8.38)$ & $(6.58)$ & $(6.74)$ & $(8.14)$ \\
\hline \multirow[t]{2}{*}{$\mathbf{T}_{2}$} & 3.66 & 3.82 & 3.98 & 4.15 & 4.07 & 4.16 & 4.18 & 4.06 \\
\hline & $(12.90)$ & $(14.09)$ & $(15.34)$ & $(16.72)$ & $(16.06)$ & $(16.81)$ & $(16.97)$ & $(15.98)$ \\
\hline \multirow[t]{2}{*}{$\mathbf{T}_{3}$} & 3.70 & 3.24 & 3.33 & 2.90 & 2.88 & 2.67 & 2.62 & 2.94 \\
\hline & $(13.19)$ & $(10.00)$ & $(10.59)$ & $(7.91)$ & $(7.79)$ & $(6.63)$ & $(6.36)$ & $(8.14)$ \\
\hline \multirow[t]{2}{*}{$\mathbf{T}_{4}$} & 3.79 & 3.84 & 3.87 & 3.90 & 3.98 & 4.05 & 4.16 & 3.97 \\
\hline & $(13.86)$ & $(14.25)$ & (14.48) & $(14.71)$ & (15.34) & (15.90) & $(16.81)$ & $(15.26)$ \\
\hline \multirow[t]{2}{*}{$\mathbf{T}_{5}$} & 3.56 & 3.71 & 3.93 & 4.06 & 3.92 & 3.97 & 4.09 & 3.95 \\
\hline & (12.17) & (13.26) & (14.94) & (15.98) & (14.87) & $(15.26)$ & (16.23) & $(15.10)$ \\
\hline \multirow[t]{2}{*}{$\mathbf{T}_{6}$} & 3.58 & 2.95 & 3.08 & 2.44 & 2.69 & 2.19 & 2.36 & 2.62 \\
\hline & $(12.32)$ & $(8.20)$ & (8.99) & $(5.45)$ & (6.74) & $(4.30)$ & $(5.07)$ & $(6.36)$ \\
\hline \multirow[t]{2}{*}{$\mathbf{T}_{7}$} & 3.93 & 4.37 & 4.27 & 4.14 & 4.24 & 4.25 & 4.39 & 4.28 \\
\hline & (14.94) & (18.60) & (17.73) & (16.64) & (17.48) & (17.56) & (18.77) & $(17.82)$ \\
\hline S. Em $\pm \mathbf{T}$ & 0.22 & 0.18 & 0.19 & 0.19 & 0.18 & 0.13 & 0.17 & 0.08 \\
\hline $\mathbf{Y} \times \mathbf{T}$ & - & - & - & - & - & - & - & 0.18 \\
\hline C.D. at $5 \% \mathrm{~T}$ & NS & 0.56 & 0.59 & 0.60 & 0.56 & 0.42 & 0.53 & 0.23 \\
\hline $\mathbf{Y} \times \mathbf{T}$ & - & - & - & - & - & - & - & NS \\
\hline C. V. (\%) & 10.64 & 8.91 & 9.14 & 9.90 & 9.06 & 7.00 & 8.76 & 8.86 \\
\hline \multicolumn{8}{|c|}{ * Figures in parentheses are retransformed values; those outsidoare } & led values \\
\hline
\end{tabular}

Table.4 Effectiveness of different entomopathogenic fungi against mango hoppers (2013-14)

\begin{tabular}{|c|c|c|c|c|c|c|c|c|}
\hline \multirow[t]{3}{*}{ Treatments } & \multicolumn{8}{|c|}{ No. of mango hoppers/twig } \\
\hline & \multirow{2}{*}{$\begin{array}{c}\text { Before } \\
\text { spray }\end{array}$} & \multicolumn{2}{|c|}{$1^{\text {st }}$ Spray } & \multicolumn{2}{|c|}{$2^{\text {nd }}$ Spray } & \multicolumn{2}{|c|}{$3^{\text {rd }}$ Spray } & \multirow{2}{*}{$\begin{array}{c}\text { Pooled over period } \\
\text { over sprays }\end{array}$} \\
\hline & & 7 DAS & 14 DAS & 7 DAS & 14 DAS & 7 DAS & 14 DAS & \\
\hline \multirow[t]{2}{*}{$\mathbf{T}_{1}$} & 3.71 & 3.32 & 2.79 & 3.08 & 3.08 & 2.73 & 2.87 & 2.98 \\
\hline & $(13.26)$ & $(10.52)$ & $(7.28)$ & $(8.99)$ & $(8.99)$ & $(6.95)$ & $(7.74)$ & $(8.38)$ \\
\hline \multirow[t]{2}{*}{$\mathbf{T}_{2}$} & 3.84 & 3.77 & 3.86 & 3.77 & 3.84 & 3.93 & 3.94 & 3.85 \\
\hline & $(14.25)$ & $(13.71)$ & $(14.40)$ & $(13.71)$ & $(14.25)$ & $(14.94)$ & $(15.02)$ & $(14.32)$ \\
\hline \multirow[t]{2}{*}{$\mathbf{T}_{3}$} & 3.72 & 3.33 & 2.80 & 3.08 & 3.07 & 2.75 & 2.85 & 2.98 \\
\hline & $(13.34)$ & $(10.59)$ & $(7.34)$ & $(8.99)$ & $(8.92)$ & $(7.06)$ & $(7.62)$ & (8.38) \\
\hline \multirow[t]{2}{*}{$\mathbf{T}_{4}$} & 3.92 & 3.80 & 3.82 & 3.72 & 3.72 & 3.94 & 3.83 & 3.81 \\
\hline & (14.87) & (13.94) & (14.09) & (13.34) & (13.34) & (15.02) & (14.17) & (14.02) \\
\hline \multirow[t]{2}{*}{$\mathbf{T}_{5}$} & 3.80 & 3.68 & 3.82 & 3.69 & 3.86 & 3.96 & 3.95 & 3.83 \\
\hline & (13.94) & (13.04) & (14.09) & (13.12) & (14.40) & (15.18) & (15.10) & (14.17) \\
\hline \multirow[t]{2}{*}{$\mathbf{T}_{6}$} & 3.70 & 2.32 & 2.02 & 2.13 & 2.18 & 2.08 & 2.05 & 2.13 \\
\hline & (13.19) & $(4.88)$ & $(3.58)$ & $(4.04)$ & $(4.25)$ & $(3.83)$ & $(3.70)$ & $(4.04)$ \\
\hline \multirow[t]{2}{*}{$\mathbf{T}_{7}$} & 3.95 & 4.36 & 4.35 & 4.13 & 4.26 & 4.22 & 4.38 & 4.29 \\
\hline & (15.10) & (18.51) & (18.42) & (16.56) & $(17.65)$ & $(17.31)$ & (18.68) & (17.90) \\
\hline
\end{tabular}




\begin{tabular}{|c|c|c|c|c|c|c|c|c|}
\hline S. $\mathbf{E m} \pm \mathbf{T}$ & 0.27 & 0.25 & 0.25 & 0.22 & 0.23 & 0.20 & 0.26 & 0.10 \\
\hline $\mathbf{Y} \times \mathbf{T}$ & - & - & - & - & - & - & - & 0.18 \\
\hline C.D. at 5\% $\mathrm{T}$ & NS & 0.77 & 0.76 & 0.65 & 0.71 & 0.63 & 0.80 & 0.29 \\
\hline $\mathbf{Y} \times \mathbf{T}$ & - & - & - & - & - & - & - & NS \\
\hline C. V. (\%) & 12.50 & 12.46 & 12.90 & 11.09 & 11.88 & 10.75 & 13.40 & 12.61 \\
\hline \multicolumn{9}{|c|}{ * Figures in parentheses are retransformed values; those outsidoare $\quad$ transformed values } \\
\hline
\end{tabular}

Table.5 Effectiveness of different entomopathogenic fungi against mango hoppers (Pooled over years)

\begin{tabular}{|c|c|c|c|c|c|c|c|c|}
\hline \multirow[t]{2}{*}{ Treatments } & \multicolumn{8}{|c|}{ No. of mango hoppers/ twig } \\
\hline & \multicolumn{2}{|c|}{ 2011-12 } & \multicolumn{2}{|r|}{$2012-13$} & \multicolumn{2}{|c|}{ 2013-14 } & \multicolumn{2}{|c|}{ Pooled } \\
\hline $\mathbf{T}_{1}$ & \multicolumn{2}{|c|}{$\begin{array}{l}2.58^{\mathrm{b}} \\
(6.18)\end{array}$} & \multicolumn{2}{|c|}{$\begin{array}{l}2.94^{\mathrm{b}} \\
(8.14)\end{array}$} & \multicolumn{2}{|c|}{$\begin{array}{l}2.98^{\mathrm{b}} \\
(8.38)\end{array}$} & \multicolumn{2}{|c|}{$\begin{array}{l}2.83^{\mathrm{b}} \\
(7.51)\end{array}$} \\
\hline $\mathbf{T}_{2}$ & \multicolumn{2}{|c|}{$\begin{array}{c}3.79^{c} \\
(13.86)\end{array}$} & \multicolumn{2}{|r|}{$\begin{array}{l}4.06^{\mathrm{cd}} \\
(15.98)\end{array}$} & \multicolumn{2}{|c|}{$\begin{array}{c}3.85^{\mathrm{c}} \\
(14.32)\end{array}$} & \multicolumn{2}{|c|}{$\begin{array}{c}3.90^{\mathrm{c}} \\
(14.71)\end{array}$} \\
\hline $\mathbf{T}_{3}$ & \multicolumn{2}{|c|}{$\begin{array}{l}2.53^{\mathrm{ab}} \\
(5.90)\end{array}$} & \multicolumn{2}{|r|}{$\begin{array}{l}2.94^{\mathrm{b}} \\
(8.14)\end{array}$} & \multicolumn{2}{|c|}{$\begin{array}{l}2.98^{\mathrm{b}} \\
(8.38)\end{array}$} & \multicolumn{2}{|c|}{$\begin{array}{l}2.82^{\mathrm{b}} \\
(7.45)\end{array}$} \\
\hline $\mathbf{T}_{4}$ & \multicolumn{2}{|c|}{$\begin{array}{c}3.67^{\mathrm{c}} \\
(12.97)\end{array}$} & \multicolumn{2}{|r|}{$\begin{array}{c}3.97^{\mathrm{c}} \\
(15.26)\end{array}$} & \multicolumn{2}{|c|}{$\begin{array}{c}3.81^{\mathrm{c}} \\
(14.02)\end{array}$} & \multicolumn{2}{|c|}{$\begin{array}{c}3.82^{\mathrm{c}} \\
(14.09)\end{array}$} \\
\hline $\mathbf{T}_{5}$ & \multicolumn{2}{|c|}{$\begin{array}{c}3.64^{c} \\
(12.75)\end{array}$} & \multicolumn{2}{|r|}{$\begin{array}{c}3.95^{\mathrm{c}} \\
(15.10)\end{array}$} & \multicolumn{2}{|c|}{$\begin{array}{c}3.83^{\mathrm{c}} \\
(14.17)\end{array}$} & \multicolumn{2}{|c|}{$\begin{array}{c}3.81^{\mathrm{c}} \\
(14.02)\end{array}$} \\
\hline $\mathbf{T}_{6}$ & \multicolumn{2}{|c|}{$\begin{array}{l}2.25^{\mathrm{a}} \\
(4.56)\end{array}$} & \multicolumn{2}{|r|}{$\begin{array}{l}2.62^{\mathrm{a}} \\
(6.36)\end{array}$} & \multicolumn{2}{|c|}{$\begin{array}{r}2.13^{\mathrm{a}} \\
(4.04)\end{array}$} & \multicolumn{2}{|c|}{$\begin{array}{l}2.33^{\mathrm{a}} \\
(4.93)\end{array}$} \\
\hline $\mathbf{T}_{7}$ & \multicolumn{2}{|c|}{$\begin{array}{c}3.93^{\mathrm{c}} \\
(14.94)\end{array}$} & & $\begin{array}{l}4.28^{\mathrm{d}} \\
(17.82)\end{array}$ & & $\begin{array}{l}29^{d} \\
.90)\end{array}$ & & $\begin{array}{l}.16^{\mathrm{d}} \\
5.81)\end{array}$ \\
\hline & $\begin{array}{l}\text { S. } \\
\text { Em. } \pm\end{array}$ & $\begin{array}{l}\text { C. D. } \\
\text { at } 5 \%\end{array}$ & $\begin{array}{l}\text { S. } \\
\text { Em. } \pm\end{array}$ & C. D. at $5 \%$ & $\begin{array}{l}\text { S. } \\
\text { Em. } \pm\end{array}$ & $\begin{array}{l}\text { C. D. at } \\
5 \%\end{array}$ & S. Em. \pm & $\begin{array}{l}\text { C. D. at } \\
5 \%\end{array}$ \\
\hline Treatments (T) & 0.10 & 0.27 & $0.0 \overline{8}$ & 0.23 & 0.09 & 0.26 & 0.05 & 0.14 \\
\hline Years $(Y)$ & - & - & - & - & - & - & 0.03 & 0.09 \\
\hline Sprays (S) & 0.03 & 0.10 & 0.03 & 0.08 & 0.05 & NS & 0.03 & 0.09 \\
\hline Periods (P) & 0.03 & 0.08 & 0.02 & 0.07 & 0.04 & NS & 0.03 & NS \\
\hline $\mathbf{T} \times \mathbf{Y}$ & - & - & - & - & - & - & 0.09 & NS \\
\hline $\mathbf{T} \times \mathbf{S}$ & 0.05 & 0.14 & 0.04 & 0.11 & 0.06 & NS & 0.09 & 0.24 \\
\hline $\mathbf{T} \times \mathbf{P}$ & 0.09 & NS & 0.08 & NS & 0.12 & NS & 0.07 & NS \\
\hline $\mathbf{Y} \times \mathbf{S}$ & - & - & - & - & - & - & 0.06 & NS \\
\hline$Y \times P$ & - & - & - & - & - & - & 0.05 & NS \\
\hline $\mathbf{S} \times \mathbf{P}$ & 0.07 & NS & 0.06 & NS & 0.10 & NS & 0.05 & NS \\
\hline$T \times Y \times S$ & - & - & - & - & - & - & 0.15 & NS \\
\hline$T \times Y \times P$ & - & - & - & - & - & - & 0.12 & NS \\
\hline$Y \times S \times P$ & - & - & - & - & - & - & 0.08 & NS \\
\hline $\mathbf{T} \times S \times P$ & 0.13 & NS & 0.11 & NS & 0.17 & NS & 0.12 & NS \\
\hline$T \times Y \times S \times P$ & - & - & - & - & - & - & 0.21 & NS \\
\hline C.V.\% & & & & 8.86 & & .13 & & 0.86 \\
\hline $\begin{array}{l}\text { Figures in paren } \\
\text { Figures in Letter } \\
\text { NS = Non -signi }\end{array}$ & $\begin{array}{l}\text { eses are } \\
\text { in com } \\
\text { int }\end{array}$ & $\begin{array}{l}\text { transfo } \\
\text { on are s }\end{array}$ & $\begin{array}{l}\text { led valu } \\
\text { tistically }\end{array}$ & $\begin{array}{l}\text { es; those outsi } \\
\text { at par as per }\end{array}$ & $\begin{array}{l}\text { are } \\
\text { NMRT }\end{array}$ & trans & formed va & \\
\hline
\end{tabular}


Pooled over periods and sprays data for rabi, 2012-13 indicated that the treatment of imidacloprid 17.8 SL registered significantly least (6.36 hoppers/ twig) number of hoppers compared to rest of the treatments. While among the bio-pesticides treatments, mango trees sprayed either with $V$. lecanii or $M$. anisopliaeon tree trunk during off-season + at flowering stage registered lowest (8.14 hoppers/ twig) numbers of hoppers than rest of the treatments. The treatment of alone application of $V$. lecaniior $M$. anisopliae only during off-season on tree trunk proved inferior in reducing hoppers population but registered lower hoppers than untreated control (17.82 hoppers/ twig).

\section{Year 2013-14}

Pooled over periods and sprays data computed for rabi, 2013-14 (Table 4) indicated that all the treatments proved significantly effective when compared with control after 7 and 14 days of spray as well as in pooled. The results indicated that the lowest (4.04 hoppers/ twig) numbers of hoppers were registered in the treatment of imidacloprid 17.8 SL applied on tree trunk during off-season + one spray of the same applied at flowering stage. Both the treatments of entomopathogenic fungi i.e. $V$. lecanii and $M$. anisopliae registered equal numbers of hoppers i.e. 8.38 hoppers/ twig when applied on tree trunk during off-season + during flowering stage and found effective next to chemical insecticide treatment. While, the treatment of application of $V$. lecaniior $M$. anisopliae only on the tree trunk during offseason proved inferior against mango hoppers

\section{Pooled over periods and years}

Among the different entomopathogenic fungi evaluated for their bio efficacy against mango hopper during 2011-12, 2012-13 and 2013-14 (Table 5), the data of pooled over periods over years depicted that significantly lowest number of mango hopper/twig was recorded in the treatment $\mathrm{T}_{3}$ i.e. application of $V$. lecanni (Lecanicillium lecanii) on tree trunk during off-season + at flowering period @ 5 g/ litre(7.45 hoppers/twig), which was found at par with the treatment of $\mathrm{T}_{1}$ i.e.application of M.anisopliae on tree trunk during offseason + at flowering period(7.51 hoppers/twig). While, application of $V$. lecanni (L.Lecanii) only on tree trunk during off-season@ 5 g/ litre registered lower (14.09 hoppers/twig) numbers of hoppers and it was followed by treatment of $\mathrm{T}_{2}$ i.e. application of M.anisopliae on tree trunk during only during off-season@ 5 g/ litre(14.71 hoppers/twig).

The efficacy entomopathogenic fungi against mango hoppers also get support from Valvi et al., (2018), who reported that mixed application of Leccaniicillium leccanii + Metarhizium anisopliae and sole application of Metarhizium anisopliae found effective against mango hopper. The entomopathogenic fungi $V$. leccanii effectiveness in warding off the mango hopper attack stated by Trivedi and Singh (2007) and Singh (2008). The above reports render support to the present observation of superior efficacy of fungal formulation against mango hoppers.

\section{References}

Das, G., Chavan, P. D., Sawant, B. N. 1969. The mango hoppers mainly suck the sap from tender shoots, leaves and inflorescence, Indian Journal of Plant Protection, 33:46-49.

Gangolly, S. R., Singh, R., Katyal, S. L., Singh, D. Mango, ICAR, New Delhi, 1957. 492.

Gundappa, Kamala Jayanthi P. D. and Verghese, A. 2014. Migratory behaviour of mango hopper, Idioscopus spp. in relation to host plant flowering phenology: A synchronous shift. An Int 
J Life Sci., The Bioscan,9(2): 639-641.

Singh, R. 2008. Evaluation of some biopesticides against Mango hoppers (Idioscopus clypealis and Amritodus atkinsoni) and flower visitors of mango. Indian journal of Plant Protection, 36(1):24-27.

Tandon, P. L. andVerghese, A. 1985. World list of insect, mite and other pests of mango. Technical Document No. 5, IIHR, Bangalore.
Trivedi, T. P. and Singh, A. 2007. Validation and Promotion of IPM in public Private Partnership. Crop Care., 32:7-17.

Valvi, A. S., Mohite, P. B. and Tarate, S. P. 2018. Efficacy of various entomopathogenic fungi against mangohopper (Amritodus atkinsoni Leth.). Journal of Pharmacognosy and Phytochemistry, 7(5): 1847-1851.

\section{How to cite this article:}

Raghunandan, B. L., T. B. Kapadiya, N. B. Patel, N. M. Patel and Mehta, D. M. 2020. Efficacy of Different Entomopathogenic Fungi against Mango Hoppers in Middle Gujarat. Int.J.Curr.Microbiol.App.Sci. 9(08): 2310-2316. doi: https://doi.org/10.20546/ijcmas.2020.908.265 